TITLE:

\title{
Global gyrokinetic simulation of turbulence driven by kinetic ballooning mode
}

$\operatorname{AUTHOR}(\mathrm{S})$ :

Ishizawa, A.; Imadera, K.; Nakamura, Y.; Kishimoto, Y.

\section{CITATION:}

Ishizawa, A....[et al]. Global gyrokinetic simulation of turbulence driven by kinetic ballooning mode. Physics of Plasmas 2019, 26(8): 082301.

\section{ISSUE DATE:}

2019-08

URL:

http://hdl.handle.net/2433/243340

\section{RIGHT:}

This article may be downloaded for personal use only. Any other use requires prior permission of the author and AIP Publishing. This article appeared in (citation of published article) and may be found at https://doi.org/10.1063/1.5100308.; The full-text file will be made open to the public on 6 August 2020 in accordance with publisher's 'Terms and Conditions for Self-Archiving'. 


\title{
Global gyrokinetic simulation of turbulence driven by kinetic ballooning mode
}

Cite as: Phys. Plasmas 26, 082301 (2019); doi: 10.1063/1.5100308

Submitted: 17 April 2019 - Accepted: 11 July 2019 .

Published Online: 6 August 2019

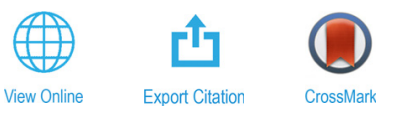

\author{
A. Ishizawa, K. Imadera, Y. Nakamura, and Y. Kishimoto
}

\section{AFFILIATIONS}

Graduate School of Energy Science, Kyoto University, Uji, Kyoto 611-0011, Japan

\begin{abstract}
Nonlinear evolution of the kinetic ballooning mode (KBM) is investigated by extending the global toroidal gyrokinetic simulation code (GKNET) to an electromagnetic regime. It is found that the saturation process of KBM, which is unstable at high normalized pressure $\beta$, is significantly different from the ion temperature gradient (ITG) mode, which is unstable at low $\beta$. The KBMs get saturated by producing zonal flows and zonal magnetic fields. The production of zonal flow is weak in the initial saturation phase of KBM, which is in contrast to the ITG mode which produces strong zonal flows in the initial saturation phase. However, strong zonal flows are produced in the subsequent evolution of KBM, and a quasisteady state of KBM turbulence is established. In addition to the zonal flows, some low toroidal number modes, which are linearly stable against the KBM, dominate the KBM turbulence. The strong zonal magnetic field is also produced by the KBM. These zonal modes regulate the KBM turbulence.
\end{abstract}

Published under license by AIP Publishing. https://doi.org/10.1063/1.5100308

\section{INTRODUCTION}

It is desirable to increase normalized pressure $\beta$ of magnetically confined plasmas because $\beta$ is linked to the fusion reaction rate and the production of the bootstrap current which is responsible for steady operation of tokamaks. Turbulence driven by drift waves becomes electromagnetic at finite $\beta$, and typical drift-wave instabilities are electromagnetic ion-temperature gradient (ITG) modes appearing at low $\beta$ and kinetic ballooning modes (KBMs) destabilized at high $\beta .^{1-5}$ The kinetic ballooning mode is a pressure gradient driven magnetohydrodynamic (MHD) instability influenced by drift waves and finite Larmor radius (FLR) effects ${ }^{6,7}$ and propagates in the ion diamagnetic direction because of its real frequency reflecting the drift-wave nature of the instability.

The electrostatic potential profile of the eigenfunction of KBM is similar to that of the ITG mode, and they commonly have a ballooning structure appearing in the bad curvature region. It is, however, understood that the nonlinear development of KBM is significantly different from that of the ITG mode. The ITG mode produces strong zonal flows, which regulate the nonlinear saturation level of ITG turbulence at low $\beta$, whereas it is hard to get a saturation of the growth of KBMs in gyrokinetic simulations because of weak zonal flows. ${ }^{2-4}$ We observe the growth of fluctuations without saturation, for instance, above $\beta_{i}$ $\approx 0.6 \%$ for the Cyclone base case (CBC) parameters except for some cases with an artificially reduced pressure gradient, ${ }^{4,8,9}$ where $\beta_{i}$ is the normalized ion pressure. This is called the run-away/nonzonal transition, ${ }^{10-12}$ and it takes place at high $\beta$ in gyrokinetic simulations using the local flux tube geometry. In these local simulations, the production of zonal flows is the main mechanism of the saturation of drift-wave instabilities, and the run-away/nonzonal transition is considered to be linked to weak zonal flows due to stochastic magnetic fields. The magnetic stochasticity is produced by magnetic reconnection, leading to the violation of magnetic surfaces. Magnetic reconnection can be caused by drift-wave instabilities at finite $\beta$ because magnetic perturbations due to the instabilities become prominent. This process of the violation of magnetic surfaces is explained in terms of parity. The ITG mode and KBM belong to the twisting/ballooning parity mode, which does not cause magnetic reconnection and retains its parity during its linear growth phase because it satisfies the linearized gyrokinetic equation. On the other hand, twisting parity modes do not satisfy the nonlinear gyrokinetic equation, ${ }^{13}$ and thus, when a twisting parity mode evolves into the nonlinear regime, i.e., its amplitude becomes finite, the free energy of the instability should be transferred to tearing parity modes through nonlinear interactions, which is called the nonlinear parity mixture. The produced tearing parity modes cause the magnetic stochasticity and influence the amplitude of ITG turbulence at finite $\beta .^{14}$ Thus, the electromagnetic drift-wave instabilities have a channel of energy transfer to tearing parity modes, which can influence the amplitude of turbulence, in addition to the production of zonal flows. The nonsaturation of instability described above takes place not only in gyrokinetic simulations but also in MHD simulations in ballooning modes. Nonlinear evolution of 
the MHD ballooning mode exhibits a growth without saturation with forming a fingerlike structure in full torus MHD simulations. ${ }^{15-17}$

We may obtain a saturation of KBMs by means of global gyrokinetic simulations because there are some mechanisms which are not included in local gyrokinetic and full torus MHD simulations. Recently, nonlinear evolution of KBM has been investigated by means of global electromagnetic gyrokinetic simulations. ${ }^{18}$ The simulation results imply the importance of the zonal mode, i.e., zonal flows, zonal magnetic fields, and zonal pressure, in the saturation of KBMs. In order to elucidate the essential mechanism of the saturation, this simulation includes only a pair of unstable KBMs with $n= \pm 10$ and $n=0$ toroidal modes. The influence of nonlinear mode coupling with other unstable and stable toroidal modes on the saturation of KBM is not understood well.

In this paper, we investigate the saturation of KBM by means of a new global electromagnetic gyrokinetic simulation code developed by extending a version of GKNET. ${ }^{19,20}$ Our simulations include nonlinear mode coupling among unstable toroidal modes and stable low toroidal modes. The original GKNET is the electrostatic full-f gyrokinetic code with the polar ${ }^{19}$ or rectangular ${ }^{20}$ coordinates in the poloidal plane. This code enables us to investigate global turbulent transport and resultant background profile formation coupled with radial mean flow shear such as the staircase ${ }^{21}$ and internal transport barrier triggered by momentum injection. ${ }^{22}$ Kinetic electron dynamics has been recently introduced to investigate electrostatic drift-wave instabilities. ${ }^{23} \mathrm{We}$ extend the code by introducing magnetic perturbations so that we are able to investigate electromagnetic drift-wave instabilities. We will present a saturation of KBM and a quasisteady state of KBM turbulence. In the quasisteady state, strong zonal flows and low toroidal modes, which are stable against KBM, dominate the fluctuations of turbulence.

The remainder of this paper is as follows. Our new simulation code is described in Sec. II. Linear and nonlinear simulation results on KBM turbulence and ITG turbulence are presented in Sec. III. Finally, the summary of results is given in Sec. IV.

\section{NUMERICAL MODEL}

We describe our new global electromagnetic simulation code, which is developed by extending GKNET,,$^{19,20}$ in this section. We divide a distribution function into the Maxwellian and perturbed parts, $F_{s}=F_{0 s}+\delta f_{s}$, where $F_{0 s}=\frac{n(r)}{\left(2 \pi T_{s}(r) / m_{s}\right)^{3 / 2}} \exp \left(-\frac{m_{s} v_{\|}^{2}}{2 T_{s}(r)}-\frac{\mu B}{T_{s}(r)}\right)$, and $\mathbf{v}=v_{\|} \mathbf{b}+\mathbf{v}_{\perp} ; \quad \mu=m_{s} v_{\perp}^{2} /(2 B)$ and $\mathbf{b}=\mathbf{B} / B$ are the magnetic moment and the unit vector of the equilibrium magnetic field, respectively. The subscript $s$ denotes the particle species such as for ions $s=i$ and electrons $s=e$. The gyrokinetic Vlasov equation for the perturbed gyrocenter distribution function, the gyrokinetic Poisson equation, and Ampère's law is

$$
\begin{aligned}
\frac{\partial \delta f_{s}}{\partial t}+ & v_{\|} \mathbf{b}_{s}^{*} \cdot \nabla \delta f_{s}-\frac{\mu}{m_{s}} \mathbf{b} \cdot \nabla B \frac{\partial \delta f_{s}}{\partial v_{\|}} \\
= & -\mathbf{v}_{d s} \cdot \nabla\left(\delta f_{s}+\frac{q_{s}}{T_{s}} F_{0 s}\langle\phi\rangle_{s}\right)-\left\langle\tilde{\mathbf{v}}_{E}\right\rangle_{s} \cdot \nabla \delta f_{s} \\
& +\frac{q_{s}}{T_{s}} F_{0 s} \mathbf{v}_{* s} \cdot \nabla\left(\langle\phi\rangle_{s}-\frac{v_{\|}}{c}\left\langle A_{\|}\right\rangle_{s}\right)-\frac{q_{s}}{m_{s}}\left\langle E_{\|}\right\rangle_{s} \frac{\partial F_{0 s}}{\partial v_{\|}},
\end{aligned}
$$

$$
\begin{gathered}
\sum_{s} q_{s} \delta n_{s}^{(p)}=0, \\
-\nabla_{\perp}^{2} A_{\|}=\frac{4 \pi}{c} \sum_{s} q_{s} \delta u_{s}^{(p)},
\end{gathered}
$$

respectively. In the equations, $\left\langle E_{\|}\right\rangle_{s}=-\mathbf{b}_{s}^{*} \cdot \nabla\langle\phi\rangle_{s}-\frac{1}{c} \frac{\partial}{\partial t}\left\langle A_{\|}\right\rangle_{s}$, $\delta n_{s}^{(p)}=\int\left\langle\delta f_{s}\right\rangle_{s} d^{3} v+\frac{q_{s} n}{T_{s}} \nabla_{\perp}^{2} \phi$, and $\delta u_{s}^{(p)}=\int v_{\|}\left\langle\delta f_{s}\right\rangle_{s} d^{3} v$ are the parallel component of the perturbed electric field, the perturbed density measured at the particle position, and the parallel component of perturbed velocity measured at the particle position, respectively. The gyroaverage for species $s$ is represented by \langle\rangle$_{s}$, and $\beta_{i}=\frac{8 \pi n_{0} T_{i 0}}{B_{0}^{2}}$ is the normalized ion pressure measured at $r=r_{0}$. It is remarked that the polarization term is approximated by the leading order term of $k_{\perp}^{2}$ in the gyrokinetic Poisson equation (2). Nonlinear terms appear in the convective derivative term caused by perturbed $\mathrm{E} \times \mathrm{B}$ flow $\left\langle\tilde{\mathbf{v}}_{E}\right\rangle_{s}$. $\nabla f=\frac{c}{B}\left[\langle\phi\rangle_{s}, f\right]$ and in the parallel component of the spatial gradient $\mathbf{b}_{s}^{*} \cdot \nabla f=\mathbf{b} \cdot \nabla f+\langle\tilde{\mathbf{b}}\rangle_{s} \cdot \nabla f=\mathbf{b} \cdot \nabla f-\frac{1}{B}\left[\left\langle A_{\|}\right\rangle_{s}, f\right]$, where $[f, g]$ $=\mathbf{b} \cdot \nabla f \times \nabla g$ is the Poisson bracket. The unit vector along the magnetic field is $\mathbf{b}_{s}^{*}=\mathbf{b}+\langle\tilde{\mathbf{b}}\rangle_{s}$, and $\langle\tilde{\mathbf{b}}\rangle_{s}=-\mathbf{b} \times \nabla\left\langle A_{\|}\right\rangle_{s}$ is the perturbed part of the magnetic field. The magnetic drift, the diamagnetic drift, and the perturbed $\mathrm{E} \times \mathrm{B}$ drift velocities are $\mathbf{v}_{d s}=\frac{c}{q_{s} B} \mathbf{b} \times(\mu \nabla B$ $\left.+m_{s} v_{\|}^{2} \mathbf{b} \cdot \nabla \mathbf{b}\right), \mathbf{v}_{* s}=\frac{c T_{s}}{q_{s} B} \mathbf{b} \times \nabla \ln F_{0 s}$, and $\left\langle\tilde{\mathbf{v}}_{E}\right\rangle_{s}=-\frac{c}{B} \nabla\langle\phi\rangle_{s} \times \mathbf{b}$, respectively.

The set of equations, Eqs. (1)-(3), is solved by extending a version of GKNET. ${ }^{19,20}$ We use the toroidal coordinates $(r, \theta, \varphi)$ in the configuration space and $\left(v_{\|}, \mu\right)$ in the velocity space. In our numerical simulation code, the time integration is made by the fourth-order Runge-Kutta method. The derivatives with respect to $r, \theta$, and $v_{\|}$are approximated by the fourth-order finite difference method, and the Fourier expansion is used along the toroidal direction $f\left(r, \theta, \varphi, v_{\|}, \mu, t\right)=\sum_{n} f_{n}\left(r, \theta, v_{\|}, \mu, t\right) \exp (-i n \varphi)$. The nonlinear terms, which are expressed in terms of the Poisson bracket, are evaluated by using the fourth-order Morinishi scheme. The gyroaverage is evaluated by using the Pade approximation $\langle f\rangle_{s} \approx \frac{f}{1+\rho_{i}^{2} k_{\perp}^{2} / 4}$, leading to an FLR equation $\left[1-\frac{\rho_{i}^{2}}{4}\left(\frac{\partial^{2}}{\partial r^{2}}-\frac{m^{2}}{r^{2}}\right)\right]\langle f\rangle_{s}=f$, where $m$ is the poloidal wavenumber. It is remarked that the FLR effects of electrons are neglected, i.e., $\langle f\rangle_{e}=f$. The FLR equation, the Poisson equation, and Ampère's law are solved by using the Fourier expansion along the poloidal direction. When we calculate the integral over the velocity space in the Poisson equation and Ampère's law, the rectangular integration is used along the $v_{\|}$direction and the Gauss-Legendre method is used along the $\mu$ direction.

Numerical simulations are carried out for the Cyclone base case (CBC) like equilibrium so that $\mathbf{v}_{d s} \cdot \nabla=-\frac{c}{q_{s} B} \frac{m_{s} v_{\|}^{2}+\mu B}{R_{0}}\left(\sin \theta \frac{\partial}{\partial r}\right.$ $\left.+\cos \theta \frac{\partial}{r \partial \theta}\right), \quad \mathbf{V}_{* s} \cdot \nabla=\frac{c T_{s}}{q_{s} B F_{0 s}}\left[\frac{\partial F_{0 s}}{\partial r} \frac{\partial}{r \partial \theta}-\frac{\partial F_{0 s}}{r \partial \theta} \frac{\partial}{\partial r}\right], \quad q(r / a)=0.85+2.18$ $(r / a)^{2}, n(r)=n_{0} \exp \left[-\frac{\delta_{r}}{L_{n}} \tanh \left(\frac{r-r_{0}}{\delta_{r}}\right)\right], \quad$ and $\quad T_{s}(r)=T_{0 s} \exp \left[-\frac{\delta_{r}}{L_{T s}}\right.$ $\left.\tanh \left(\frac{r-r_{0}}{\delta_{r}}\right)\right]$, with $r_{0}=a / 2, \delta_{r}=0.3 a, R_{0} / L_{T s}=6.66$, and $R_{0} / L_{n}=2.22$. Figure 1 shows the density, temperature, and safety factor profiles. We set the inverse of the aspect ratio $a / R_{0}=0.36$ and $\rho * \equiv \rho_{T i} / a=1 / 100$, where the leading order of Larmor radius $\rho_{i}=v_{\perp} / \Omega_{i}$ is written as $\rho_{T i}$ $=v_{T i} / \Omega_{i}$ and $v_{T i}=\sqrt{T_{0 i} / m_{i}}$ is the ion thermal velocity. The mass ratio is set to be $m_{i} / m_{e}=100$ and 400 in linear calculations and 


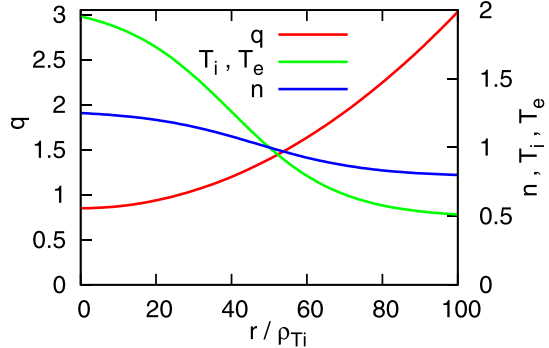

FIG. 1. Equilibrium density, temperature, and safety factor profiles.

$m_{i} / m_{e}=100$ in nonlinear calculations. The number of grid points is $\left(N_{r}, N_{\theta}, N_{v \|}, N_{\mu}\right)=(128,256,64,16)$. The toroidal modes are chosen to be even numbers $n=-30,-28, \ldots,-2,0,2,4, \ldots, 28,30$ so that the simulation domain is a half of a torus.

\section{SIMULATION RESULTS}

Here, we examine drift-wave instabilities at finite $\beta$ by solving the linearized gyrokinetic equations numerically. Figure 2 shows the linear growth rate of drift-wave instabilities as a function of $\beta_{i}$ for a toroidal mode number $n=10$. The ITG mode is unstable at low $\beta_{i}$, while the $\mathrm{KBM}$ is unstable at high $\beta_{i}$. The stabilizing effect of magnetic fluctuations on the ITG mode becomes prominent with increasing $\beta_{i}$. When $\beta_{i}$ exceeds a threshold value of $\beta_{i} \approx 1.5 \%, \mathrm{KBM}$ becomes unstable, and its growth rate increases with increasing $\beta_{i}$. The $\beta$ dependence of the growth rate for the $\mathrm{CBC}$ is similar to that obtained from another global electromagnetic gyrokinetic simulation code. ${ }^{24}$ The $\beta$ dependence of the growth rate for $m_{i} / m_{e}=400$ is similar to that for $m_{i} / m_{e}$ $=100$, but the growth rate for $m_{i} / m_{e}=400$ is a little smaller than that for $m_{i} / m_{e}=100$. It is remarked that the growth rate is time averaged for $1.3 \% \leq \beta_{i} \leq 1.5 \%$ because of a small oscillation of the growth rate. Figures 3 and 4 show the profile of the eigenfunction of the ITG mode at $\beta_{i}=0.2 \%$ and the KBM at $\beta_{i}=1.6 \%$ and $1.8 \%$ for $n=10$ on a cross section. The electrostatic potential $\phi$ profile exhibits a ballooning structure, which is the appearance of fluctuations in the bad curvature region, for both the ITG mode and the KBM in Fig. 3. A small tilting angle of the mode with respect to the midplane, referred to as the ballooning and/or Bloch angle, is observed in both the ITG mode and the $\mathrm{KBM}$, which results from the global profile effect. ${ }^{25}$ The profile of

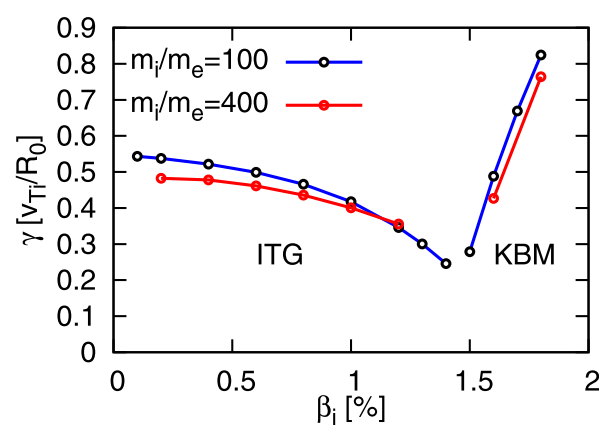

FIG. 2. Linear growth rate of drift-wave instabilities as a function of normalized ion pressure $\beta_{i}$ for the toroidal mode number, $n=10$.

the parallel component of vector potential $A_{\|}$has opposite parity to the electrostatic potential along the magnetic field line $e^{4,13}$ so that it is weak in the bad curvature region as shown in Fig. 4. Figure 5 shows the radial profile of electrostatic potential of the eigenfunction of the ITG mode at $\beta_{i}=0.2 \%$ and the $\mathrm{KBM}$ at $\beta_{i}=1.6 \%$ and $1.8 \%$. All of them have a typical ballooning mode structure which is a set of toroidally coupled poloidal modes resonating at each rational surface located at $r=r_{s}$ that satisfies $q\left(r_{s}\right)=m / n$. The ratio of the magnetic perturbation to the electrostatic potential for the ITG mode $\left|A_{\| m n}\right| /$ $\left|\phi_{m n}\right| \approx 1 / 100$ is small compared to the ratio for the KBM $\left|A_{\| m n}\right| /$ $\left|\phi_{m n}\right| \approx 1 / 10$ at $\beta_{i}=1.6 \%$ and $1.8 \%$ because $\beta_{i}=1.6 \%$ and $1.8 \%$ are larger than that of the ITG mode $\beta_{i}=0.2 \%$ which is close to the electrostatic limit $\beta_{i}=0$. This suggests that magnetic perturbations are expected to play an important role in the nonlinear saturation process of the KBM. It is noted that trapped electron modes (TEMs) appear when the ion temperature gradient is reduced from $L_{T i}\left(r_{0}\right)=6.66$ to $L_{T i}\left(r_{0}\right)=2.22$ as shown in the Appendix.

Next, we present nonlinear simulation results on turbulence driven by the $\mathrm{KBM}$ at $\beta_{i}=1.6 \%, 1.7 \%$, and $1.8 \%$ and compare them with ITG turbulence at $\beta_{i}=0.2 \%$. Figure 6 shows the time evolution of the amplitude of electrostatic potential of ITG turbulence and KBM turbulence for each toroidal mode number, $\left\langle\left|\phi_{n}\right|\right\rangle \equiv \frac{1}{V} \int\left|\phi_{n}\right| d V$ $\left[T_{i} / q_{i}\right]$. The most unstable ITG mode with $n=14$ dominantly grows in the linear growth phase $t \approx 15\left[R_{0} / v_{T i}\right]$, and then, it gets saturated by producing zonal flows $(n=0)$ at $t \approx 20$ as shown in Fig. 6(a). The
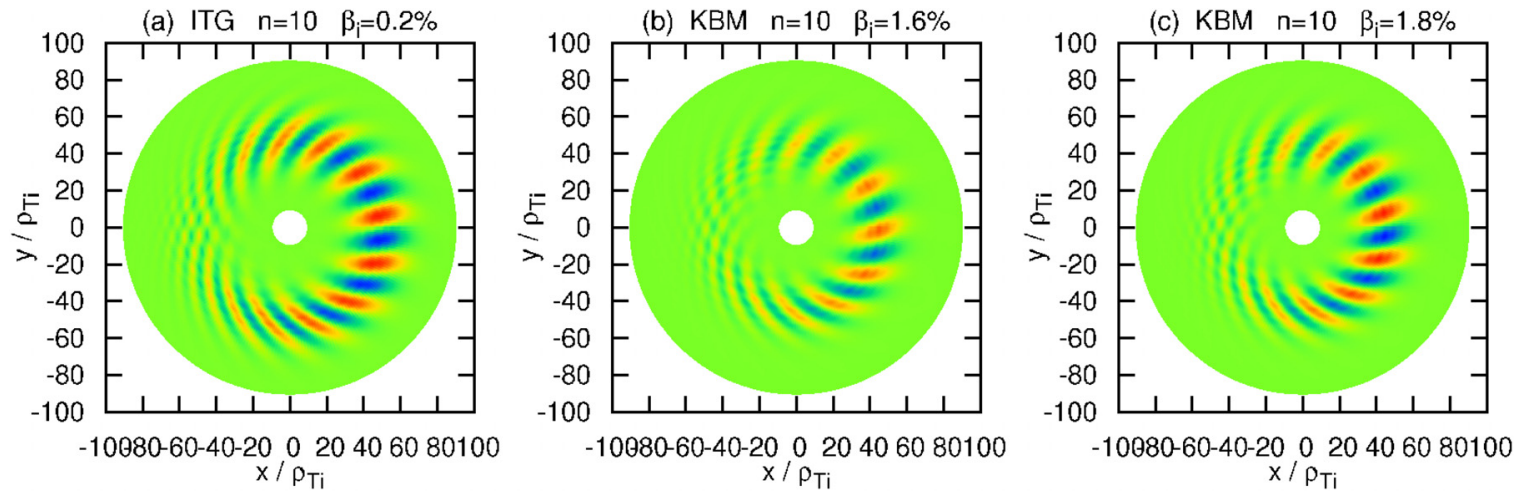

FIG. 3. Color map of electrostatic potential $\phi$ of the eigenfunction for the $n=10$ mode: (a) ITG mode at $\beta_{i}=0.2 \%$, (b) KBM at $\beta_{i}=1.6 \%$, and (c) KBM at $\beta_{i}=1.8 \%$. 
(a) ITG $\mathrm{n}=10 \quad \beta_{\mathrm{i}}=0.2 \%$

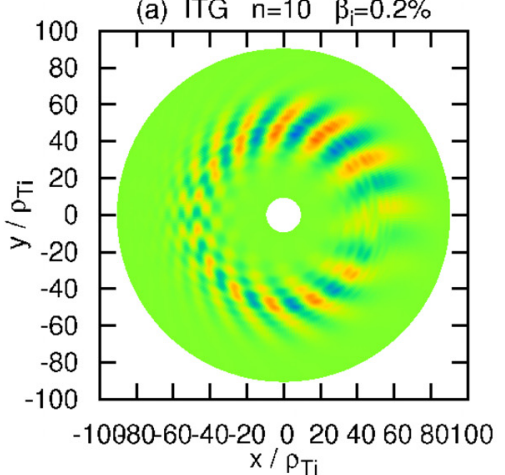

(b) KBM $n=10 \quad \beta_{1}=1.6 \%$

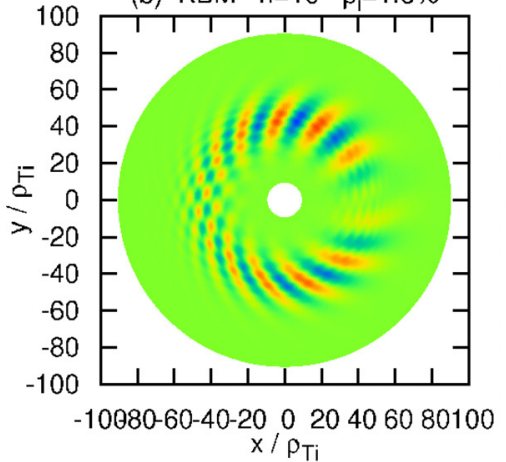

(c) KBM $\quad \mathrm{n}=10 \quad \beta_{\mathrm{i}}=1.8 \%$

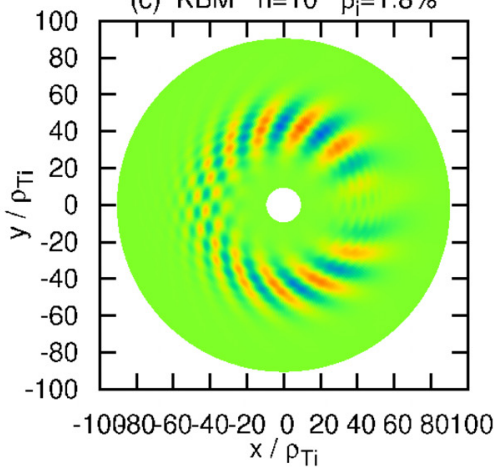

FIG. 4. Color map of the parallel component of vector potential $A_{\|}$of the eigenfunction for the $n=10$ mode: (a) ITG mode at $\beta_{i}=0.2 \%$, (b) KBM at $\beta_{i}=1.6 \%$, and (c) KBM at $\beta_{i}=1.8 \%$.

produced zonal flow is strong, and its amplitude is larger than the $n=14$ ITG mode when the ITG mode starts to get saturated at $t \approx 20$. In the quasisteady state, the zonal flows dominate the turbulence. On the other hand, the most unstable KBMs with $n=8$ and 10 dominate in the linear growth phase at $t \approx 15$ for $\beta_{i}=1.6 \%$, at $t \approx 12$ for $\beta_{i}$ $=1.7 \%$, and at $t \approx 10$ for $\beta_{i}=1.8 \%$ in Figs. $6(\mathrm{~b})-6(\mathrm{~d})$, respectively. Then, the KBMs begin to saturate at $t \approx 23$ for $\beta_{i}=1.6 \%$, at $t \approx 18$ for $\beta_{i}=1.7 \%$, and at $t \approx 14$ for $\beta_{i}=1.8 \%$. At the beginning of the saturation of the linear growth, the zonal flow production is weak so that the amplitude of zonal flow is much smaller than the KBM at $t \approx 23$ for $\beta_{i}$ $=1.6 \%$, at $t \approx 18$ for $\beta_{i}=1.7 \%$, and at $t \approx 14$ for $\beta_{i}=1.8 \%$. This is expected because the production of zonal flows by the Reynolds stress is counteracted by the Maxwell stress in nonlinear evolution of electromagnetic instabilities. ${ }^{26}$ Subsequently, other toroidal modes grow and produce zonal flows, and then, the growth of the KBM gets saturated at $t \approx 30$ for $\beta_{i}=1.6 \%$, at $t \approx 25$ for $\beta_{i}=1.7 \%$, and at $t \approx 18$ for $\beta_{i}$ $=1.8 \%$, and a quasisteady state of the KBM turbulence is eventually established at $t>40$. In the steady state of the KBM turbulence with $\beta_{i}$ $=1.6 \%, 1.7 \%$, and $1.8 \%$, zonal flows indicated by $n=0$ are comparable with those in the ITG turbulence with $\beta_{i}=0.2 \%$. It is noted that low $n$ modes such as the $n=2$ mode, which are linearly stable against the KBM, are excited by nonlinear mode coupling. Figures 7-10 show
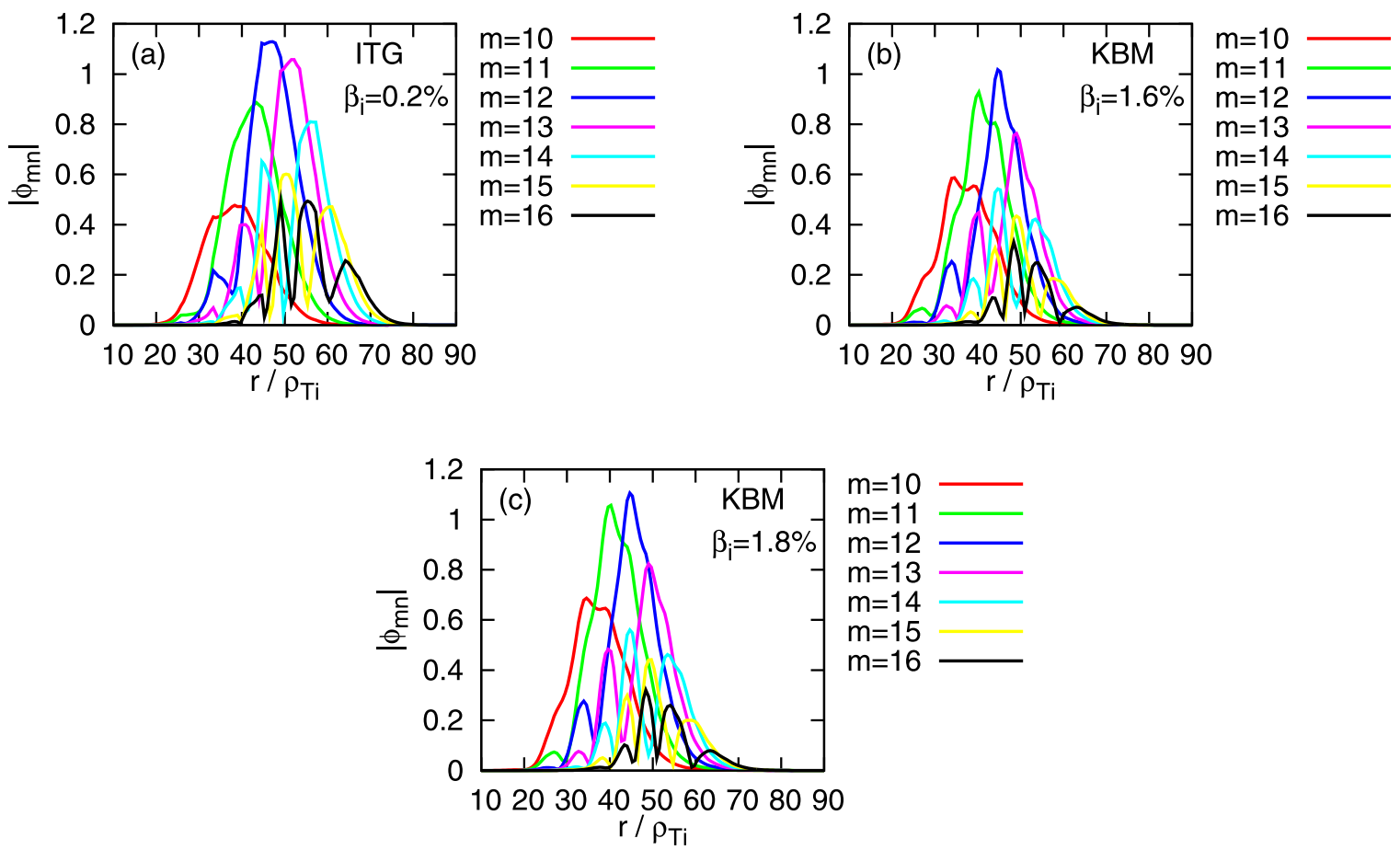

FIG. 5. Radial profile of electrostatic potential of the eigenfunction $\left|\phi_{m n}(r)\right|$ for the $n=10$ mode: (a) ITG mode at $\beta_{i}=0.2 \%$, (b) KBM at $\beta_{i}=1.6 \%$, and (c) KBM at $\beta_{i}=1.8 \%$. 

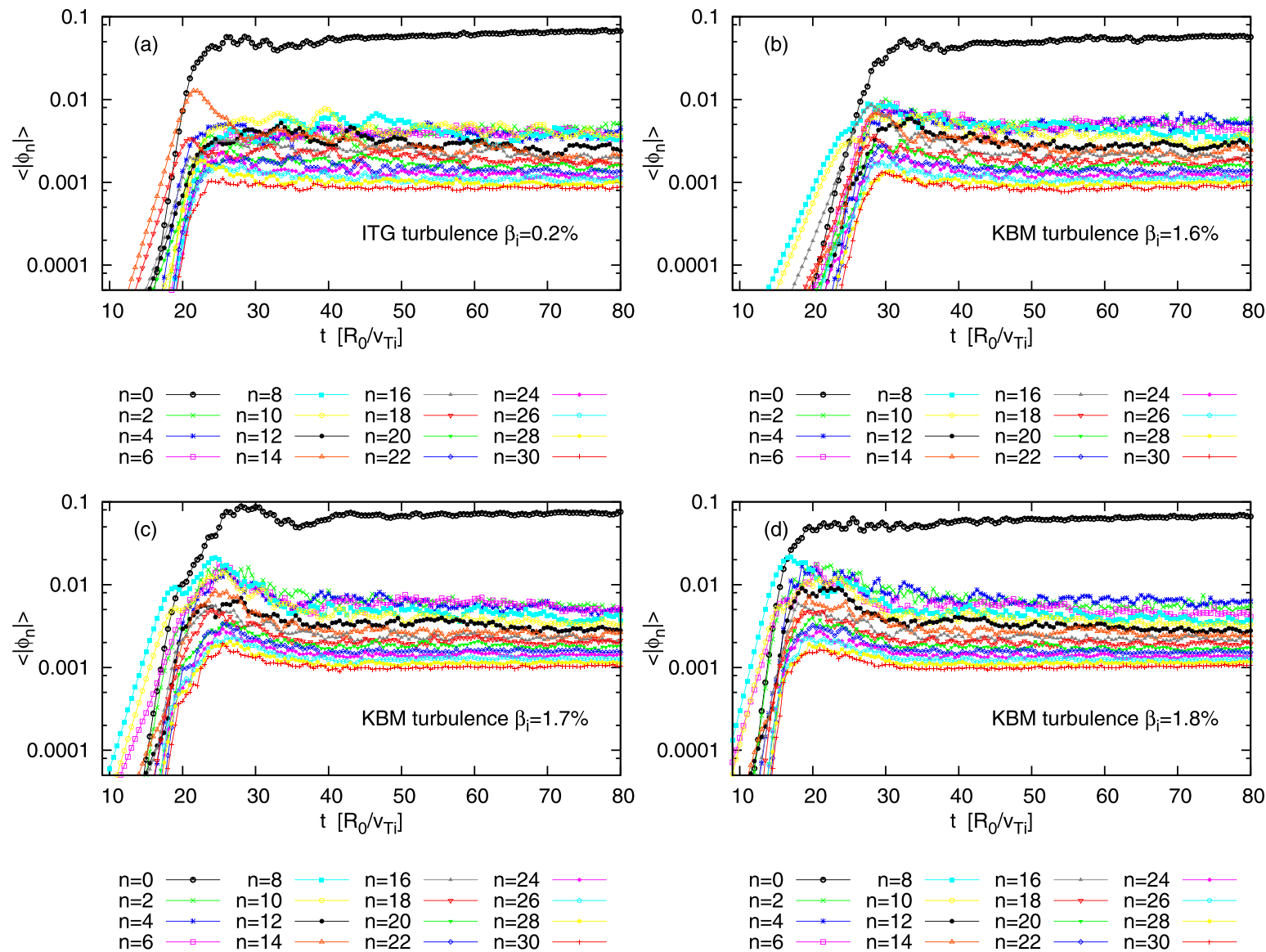

FIG. 6. Time evolution of electrostatic potential for each toroidal mode number $\left\langle\left|\phi_{n}\right|\right\rangle \equiv \frac{1}{V} \int\left|\phi_{n}\right| d V$ : (a) ITG turbulence at $\beta_{i}=0.2 \%$, (b) KBM turbulence at $\beta_{i}=1.6 \%$, (c) KBM turbulence at $\beta_{i}=1.7 \%$, and (d) KBM turbulence at $\beta_{i}=1.8 \%$.

color maps of fluctuations of the ITG turbulence and the KBM turbulence in a cross section at $t=44$ in the quasisteady state. In these figures, the turbulent fluctuations are extended in the radial direction compared to the linear growth phase shown in Fig. 3. Figure 7 shows the electrostatic potential profile $\phi(\mathbf{x})$ of ITG turbulence: $n \neq 0$ components $\sum_{n \neq 0} \phi_{n}(\mathbf{x})$, the zonal component $(n=0) \phi_{n=0}(\mathbf{x})$, and $n=14$ component $\phi_{n=14}(\mathbf{x})$, which is the most unstable ITG mode. The profile of $\sum_{n \neq 0} \phi_{n}(\mathbf{x})$ presents fully developed ITG turbulence, and the most unstable ITG mode $n=14$ is sheared by the zonal flows represented by the profile of the $n=0$ component. Figures $8-10$ show
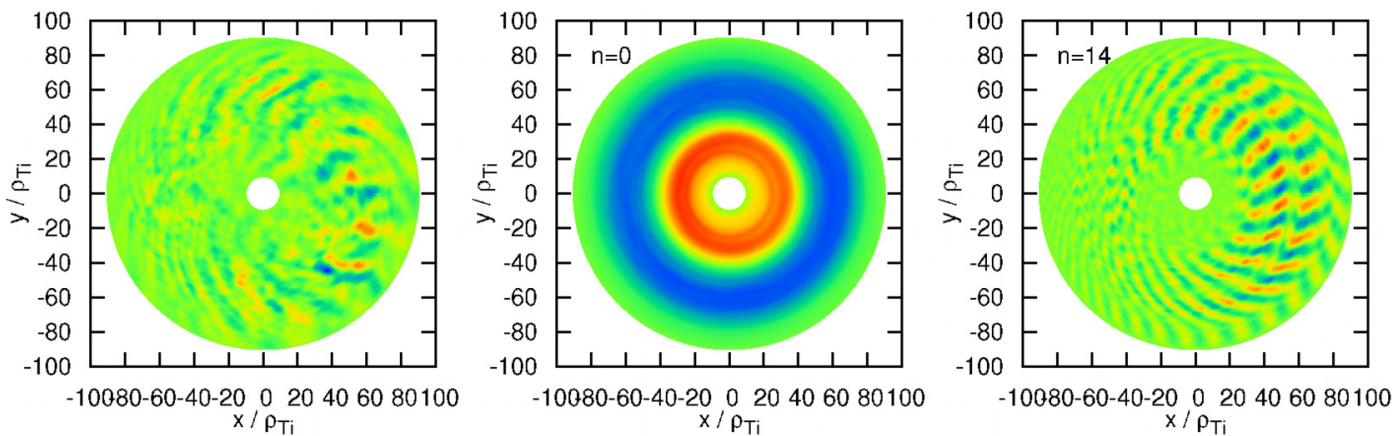

FIG. 7. Color maps of electrostatic potential $\phi(\mathbf{x})$ of ITG turbulence: $N \neq 0$ components $\sum_{n \neq 0} \phi_{n}(\mathbf{x})$, the zonal component $(n=0) \phi_{n=0}(\mathbf{x})$, and the most unstable ITG mode $(n=14) \phi_{n=14}(\mathbf{x})$ for $\beta_{i}=0.2 \%$ at $t=44$. 

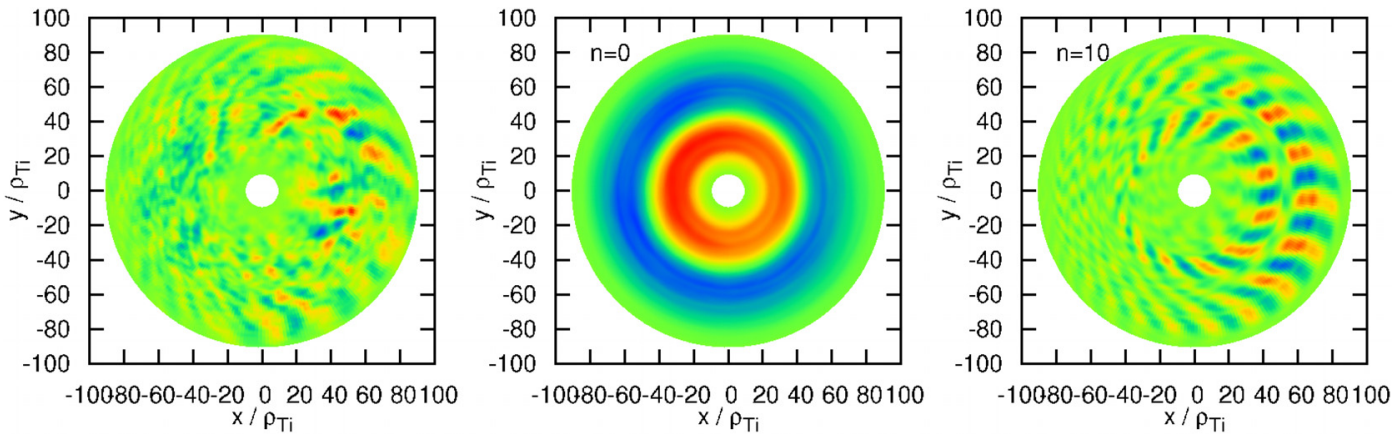

FIG. 8. Color maps of electrostatic potential $\phi(\mathbf{x})$ of KBM turbulence: $N \neq 0$ components $\sum_{n \neq 0} \phi_{n}(\mathbf{x})$, the zonal component $(n=0) \phi_{n=0}(\mathbf{x})$, and the most unstable KBM $(n=10) \phi_{n=10}(\mathbf{x})$ for $\beta_{i}=1.6 \%$ at $t=44$.
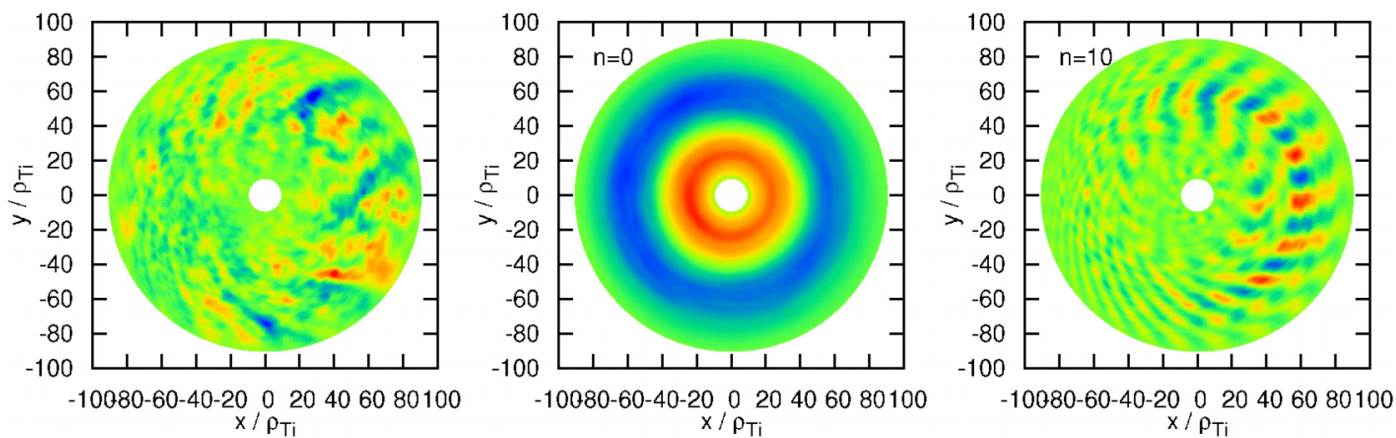

FIG. 9. Color maps of electrostatic potential $\phi(\mathbf{x})$ of KBM turbulence: $N \neq 0$ components $\sum_{n \neq 0} \phi_{n}(\mathbf{x})$, the zonal component $(n=0) \phi_{n=0}(\mathbf{x})$, and the KBM $(n=10)$ $\phi_{n=10}(\mathbf{x})$ for $\beta_{i}=1.7 \%$ at $t=44$.
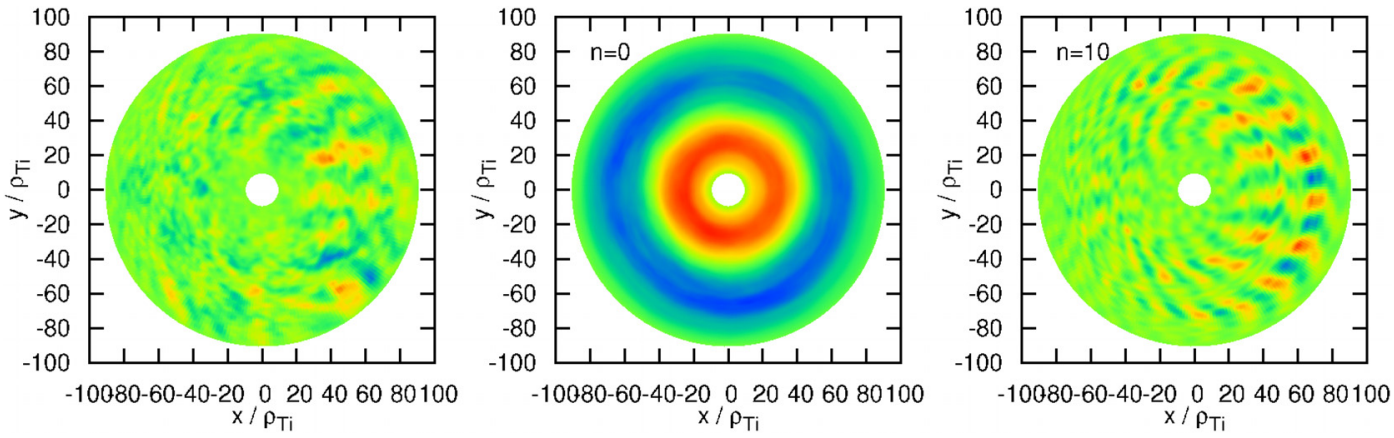

FIG. 10. Color maps of electrostatic potential $\phi(\mathbf{x})$ of KBM turbulence: $N \neq 0$ components $\sum_{n \neq 0} \phi_{n}(\mathbf{x})$, the zonal component $(n=0) \phi_{n=0}(\mathbf{x})$, and the KBM $(n=10)$ $\phi_{n=10}(\mathbf{x})$ for $\beta_{i}=1.8 \%$ at $t=44$.

the electrostatic potential profile $\phi(\mathbf{x})$ of $\mathrm{KBM}$ turbulence at $\beta_{i}$ $=1.6 \%, 1.7 \%$ and $1.8 \%$, respectively: $n \neq 0$ components $\sum_{n \neq 0} \phi_{n}(\mathbf{x})$, the zonal component $(n=0) \quad \phi_{n=0}(\mathbf{x})$, and $n=10$ component $\phi_{n=10}(\mathbf{x})$ that is the unstable KBM. The spatial profile of KBM turbulence seems to be similar to the ITG turbulence, and the unstable $\mathrm{KBM}$ with $n=10$ is sheared by the zonal flows, which are represented by the profile of $n=0$ component. The zonal flow shearing rate is evaluated to be $\bar{\gamma}_{E} \equiv \gamma_{E} R_{0} / v_{T i}=\left(k_{r} \rho_{T i}\right)^{2} \frac{q_{i}\left\langle\phi_{n=0}\right\rangle}{T_{i}} \frac{R_{0}}{\rho_{T i}}$. The zonal electrostatic potential is evaluated to be $\frac{q_{i}\left\langle\phi_{n=0}\right\rangle}{T_{i}} \approx 0.1$ from Fig. 6, and its radial wavenumber is evaluated to be $k_{r} \rho_{T i} \approx 0.1$ from Figs. $7-10$ for both the ITG turbulence and the KBM turbulence so that the shearing rate is approximated to be $\bar{\gamma}_{E} \approx 0.3$. Thus, the shearing rate is similar to the linear growth rate of the ITG mode $\bar{\gamma} \approx 0.5$ at $\beta_{i}$ $=0.2 \%$ and the $\mathrm{KBM} \bar{\gamma} \approx 0.5$ at $\beta_{i}=1.6 \%$. On the other hand, the linear growth rate of the $\mathrm{KBM}$ is $\bar{\gamma} \approx 0.8$ at $\beta_{i}=1.8 \%$, and it is much larger than the shearing rate $\bar{\gamma}_{E} \approx 0.3$. Thus, the zonal flow shearing is 

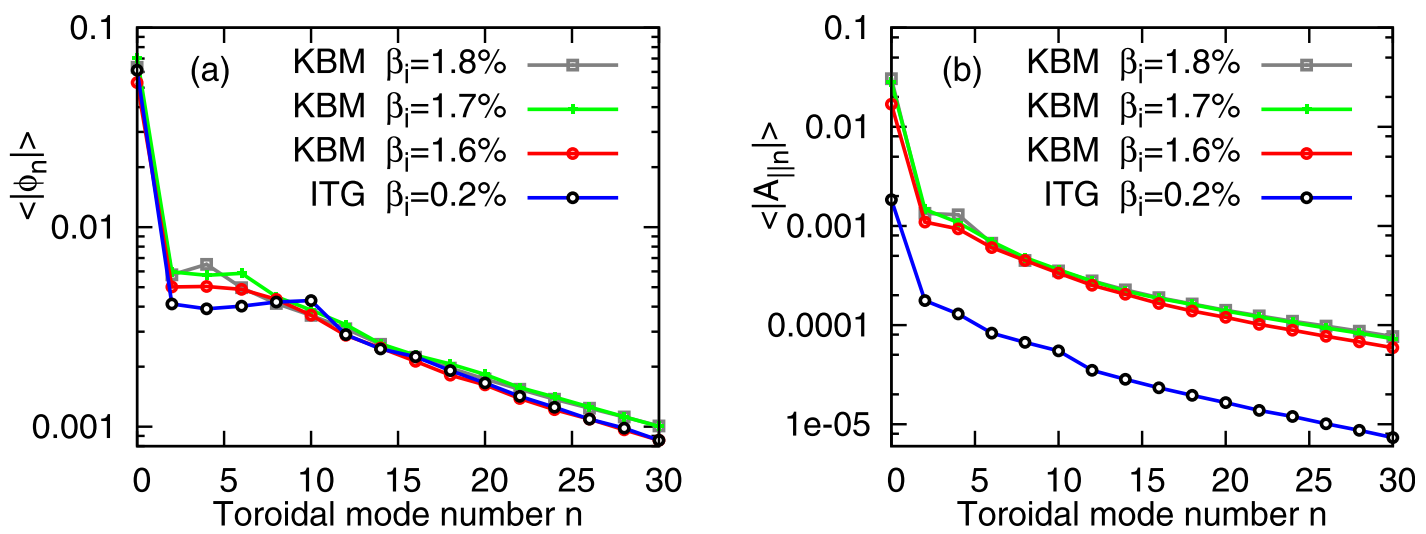

FIG. 11. Toroidal mode number spectrum of (a) the electrostatic potential $\left\langle\left|\phi_{n}\right|\right\rangle \equiv \frac{1}{V} \int\left|\phi_{n}\right| d V$ and (b) the vector potential $\left\langle\left|A_{\| n}\right|\right\rangle \equiv \frac{1}{V} \int\left|A_{\| n}\right| d V$ for ITG turbulence at $\beta_{i}$ $=0.2 \%$ and $\mathrm{KBM}$ turbulence at $\beta_{i}=1.6 \%, 1.7 \%$, and $1.8 \%$ in the quasisteady state.

not the only mechanism but other zonal modes such as the zonal magnetic field and zonal pressure can be responsible for the saturation of the KBM. In addition, the nonlinear energy transfer from the KBM to the low $n$ modes can be another saturation mechanism of the KBM.

The difference of the KBM turbulence from the ITG turbulence in the steady state is elucidated by the toroidal mode number spectra of the electrostatic and vector potential fields for $\beta_{i}=0.2 \%, 1.6 \%$, $1.7 \%$, and $1.8 \%$ as shown in Fig. 11 , where $\left\langle\left|\phi_{n}\right|\right\rangle \equiv \frac{1}{V} \int\left|\phi_{n}\right| d V$ $\left[T_{i} / q_{i}\right]$ and $\left\langle\left|A_{\| n}\right|\right\rangle \equiv \frac{1}{V} \int\left|A_{\| n}\right| d V\left[\rho_{T i} B_{0}\right]$ are averaged over a time period in the quasisteady state. The ITG turbulence has a peak at $n=10$ and is dominated by the zonal flows $(n=0)$ as shown in Fig. 11(a). On the other hand, the KBM turbulence has a broad spectrum and is dominated by the zonal flows $(n=0)$. The strong zonal flows lead to the saturation of the KBM turbulence. That is in contrast to the KBM turbulence calculated in the local flux tube geometry, ${ }^{4}$ which suffers from the run-away/nonzonal transition that is the growth of turbulent fluctuations without saturation at high $\beta$. The zonal flows are weak in the local simulations, whereas the zonal flows are strong in our global simulation. Another difference of the KBM turbulence in the global simulation from that in the local flux tube simulation is the dominance of low $n$ modes, which are not included in the local simulations. It is remarked that the amplitudes of low $n$ modes at $\beta_{i}=1.7 \%$ and $1.8 \%$ are larger than those at $\beta_{i}=1.6 \%$. Figure 11 (b) shows the spectra of the parallel component of vector potential. The zonal component and low $n$ modes dominate the magnetic perturbations for both the ITG turbulence and the KBM turbulence. The magnetic perturbations of the KBM turbulence are much larger than those of the ITG turbulence because the KBM is a kinetic MHD instability and is destabilized at higher $\beta$ than the ITG mode.

In order to clarify the influence of the zonal mode ( $n=0$ mode) on the saturation of the $\mathrm{KBM}$, we carried out a nonlinear simulation without the zonal mode for $\beta_{i}=1.7 \%$. Figure 12 shows the time evolution of the amplitude of electrostatic potential and the toroidal mode number spectrum of the electrostatic potential. The linear growth of the KBM at $t \approx 12$ is the same as that with the zonal mode in Fig. 6(c). Then, the KBMs start to get saturated at $t \approx 18$; however, they continue to grow until $t \approx 21$ in Fig. 12(a). Subsequently, low $n$ modes become dominant and get saturated after $t \approx 30$. It is not clear that the
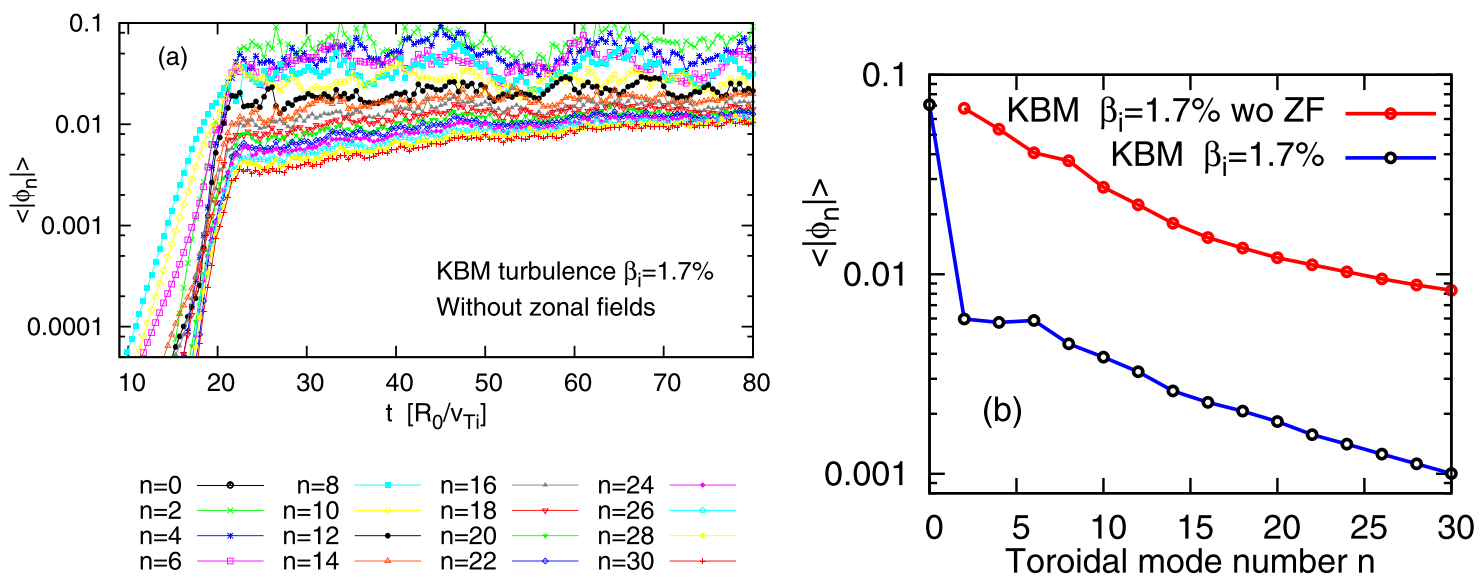

FIG. 12. Time evolution of electrostatic potential for each toroidal mode number without the zonal mode ( $n=0$ mode) (a) and the toroidal mode number spectrum of the electrostatic potential with and without the zonal mode (b) for KBM turbulence at $\beta_{i}=1.7 \%$. 


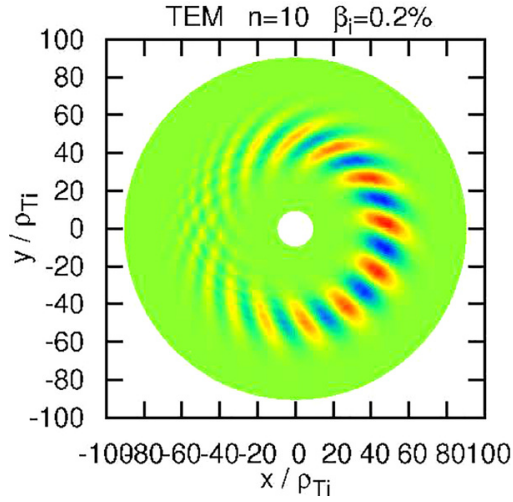

FIG. 13. Color map of electrostatic potential $\phi$ of the eigenfunction of TEM at $\beta_{i}$ $=0.2 \%$ for the $n=10$ mode.

system has reached a steady state because high $n$ modes gradually increase after $t \approx 30$. Figure 12 (b) shows that the amplitude of the KBM turbulence without the zonal mode is ten times larger than that with the zonal mode. Hence, the amplitude of the KBM turbulence is regulated by the zonal mode ( $n=0$ mode) such as zonal flow, the zonal magnetic field, and zonal pressure.

\section{SUMMARY}

We have developed a new global electromagnetic gyrokinetic simulation code by extending a version of GKNET code, which solves the electromagnetic gyrokinetic Vlasov equation together with the gyrokinetic Poisson equation and Ampère's law. Our new code enables us to investigate nonlinear evolution of KBMs and electromagnetic ITG modes. We have presented the $\beta$ dependence of the linear growth rate of drift-wave instabilities for the $\mathrm{CBC}$ like profile. The ITG mode is unstable at low $\beta$, and its growth rate decreases with increasing $\beta$ because the suppression effect of magnetic fluctuations on the ITG mode becomes prominent at finite $\beta$. On the other hand, the KBM is unstable at high $\beta$, and its growth rate increases with $\beta$.

We have obtained a saturation of the KBM and a quasisteady turbulent state in our nonlinear simulation. This is in contrast to the local flux tube calculations which exhibit the growth of fluctuations without saturation at high $\beta$. The difference from the local simulations is the production of strong zonal flows and the dominance of low $n$ modes which are stable against the KBM. We compared the nonlinear turbulent state of KBM with that of the ITG mode. The ITG turbulence is dominated by zonal flows, and the spectrum of the turbulence has a peak in the $n=10$ ITG mode. On the other hand, the KBM turbulence is dominated by zonal flows and the low toroidal modes which are linearly stable against the KBM. The spectra of the KBM turbulence exhibit higher excitation of low $n$ modes than the ITG turbulence. The mechanism of the excitation of stable modes will be investigated by evaluating nonlinear energy transfer to stable modes in the ITG and KBM turbulence in our future work.

\section{ACKNOWLEDGMENTS}

This work was supported by the Japanese Ministry of Education, Culture, Sports, Science and Technology, Grant No. 17K06991.

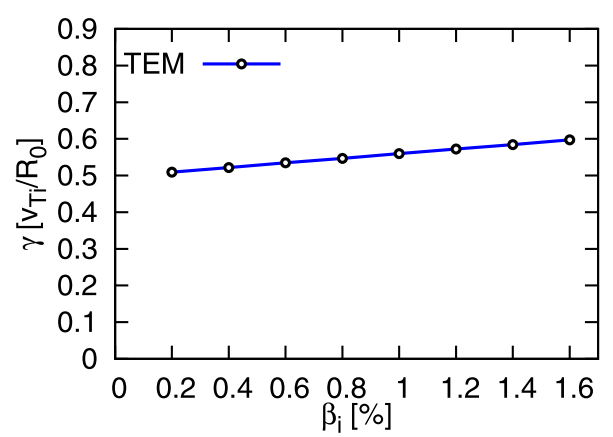

FIG. 14. Linear growth rate of TEM as a function of $\beta_{i}$ for the toroidal mode number, $n=10$.

\section{APPENDIX: TRAPPED ELECTRON MODES (TEMS)}

We have TEMs when we reduce the ion temperature gradient from $L_{T i}\left(r_{0}\right)=6.66$ to $L_{T i}\left(r_{0}\right)=2.22$. The electrostatic potential profile of the eigenfunction of TEM for $n=10$ at $\beta_{i}=0.2 \%$ is shown in Fig. 13, and it rotates in the electron diamagnetic direction. The tilting angle of the mode in the bad curvature region, i.e., the ballooning angle of the TEM, is opposite to that of the ITG mode and KBM in Fig. 3. The $\beta$ dependence of the TEM is weak as shown in Fig. 14. This weak dependence is consistent with the $\beta$ dependence of TEM presented in Ref. 27.

\section{REFERENCES}

1J. Candy, Phys. Plasmas 12, 072307 (2005).

${ }^{2}$ M. J. Pueschel, M. Kammerer, and F. Jenko, Phys. Plasmas 15, 102310 (2008).

${ }^{3}$ M. J. Pueschel and F. Jenko, Phys. Plasmas 17, 062307 (2010).

${ }^{4}$ A. Ishizawa, S. Maeyama, T.-H. Watanabe, H. Sugama, and N. Nakajima, J. Plasma Phys. 81, 435810203 (2015).

${ }^{5}$ K. Aleynikova, A. Zocco, P. Xanthopoulos, P. Helander, and C. Nuhrenberg, J. Plasma Phys. 84, 745840602 (2018).

6J. Y. Kim, W. Horton, and J. Q. Dong, Phys. Fluids B 5, 4030 (1993).

${ }^{7}$ A. Hirose, L. Zhang, and M. Elia, Phys. Rev. Lett. 72, 3993 (1994).

${ }^{8}$ A. Ishizawa, S. Maeyama, T.-H. Watanabe, H. Sugama, and N. Nakajima, Nucl. Fusion 53, 053007 (2013).

${ }^{9}$ S. Maeyama, A. Ishizawa, T.-H. Watanabe, M. Nakata, N. Miyato, M. Yagi, and Y. Idomura, Phys. Plasmas 21, 052301 (2014).

${ }^{10}$ R. E. Waltz, Phys. Plasmas 17, 072501 (2010).

${ }^{11}$ M. J. Pueschel, P. W. Terry, F. Jenko, D. R. Hatch, W. M. Nevins, T. Gorler, and D. Told, Phys. Rev. Lett. 110, 155005 (2013).

${ }^{12}$ M. J. Pueschel, P. W. Terry, and D. R. Hatch, Phys. Plasmas 21, 055901 (2014).

${ }^{13}$ A. Ishizawa, Y. Kishimoto, and Y. Nakamura, Plasma Phys. Controlled Fusion 61, 054006 (2019).

${ }^{14}$ D. R. Hatch, M. J. Pueschel, F. Jenko, W. M. Nevins, P. W. Terry, and H. Doerk, Phys. Rev. Lett. 108, 235002 (2012).

${ }^{15}$ P. Zhu, A. Bhattacharjee, and K. Germaschewski, Phys. Rev. Lett. 96, 065001 (2006).

${ }^{16}$ P. Zhu, C. C. Hegna, C. R. Sovinec, A. Bhattacharjee, and K. Germaschewski, Nucl. Fusion 49, 095009 (2009).

${ }^{17}$ P. Zhu, C. C. Hegna, and C. R. Sovinec, Phys. Rev. Lett. 102, 235003 (2009).

${ }^{18}$ G. Dong, J. Bao, A. Bhattacharjee, and Z. Lin, Phys. Plasmas 26, 010701 (2019).

${ }^{19}$ K. Imadera, Y. Kishimoto, K. Obrejan, T. Kobiki, and J. Q. Li, IAEA Fusion Energy Conference, IAEA-FEC TH/P5-8 (2014). 
${ }^{20} \mathrm{~K}$. Obrejan, K. Imadera, J. Q. Li, and Y. Kishimoto, Comput. Phys. Commun. 216, 8 (2017).

${ }^{21}$ W. Wang, Y. Kishimoto, K. Imadera, J. Q. Li, and Z. X. Wang, Nucl. Fusion 58, 056005 (2018)

${ }^{22} \mathrm{~K}$. Imadera, J. Q. Li, and Y. Kishimoto, IAEA Fusion Energy Conference, IAEA-FEC TH/P3-3 (2016)

${ }^{23}$ Z. Qin, K. Imadera, J. Q. Li, and Y. Kishimoto, Plasma Fusion Res. 13, 3403083 (2018).
${ }^{24}$ T. Gorler, X. Lapillonne, S. Brunner, T. Dannert, F. Jenko, F. Merz, and D. Told, J. Comput. Phys. 230, 7053 (2011).

${ }^{25}$ Y. Kishimoto, J.-Y. Kim, W. Horton, T. Tajima, M. J. LeBrun, and H. Shirai, Plasma Phys. Controlled Fusion 41, A663 (1999).

${ }^{26}$ V. Naulin, A. Kendl, O. E. Garcia, A. H. Nielsen, and J. J. Rasmussen, Phys. Plasmas 12, 052515 (2005).

${ }^{27}$ E. A. Belli and J. Candy, Phys. Plasmas 17, 112314 (2010). 